\title{
Responsabilidad y legitimidad en las transferencias monetarias condicionadas
}

\author{
[Responsibility and Legitimacy in Conditional Cash Transfers]
}

\author{
FACUNDO GARCÍA VALVERDE \\ Universidad de Buenos Aires \\ Consejo Nacional de Investigaciones Científicas y Técnicas \\ fgarciavalverde@gmail.com
}

\begin{abstract}
Resumen: Este artículo analiza la fundamentación normativa de un tipo de políticas de alivio contra la pobreza comunes durante los últimos años en Latinoamérica, a saber, las transferencias monetarias condicionadas. Se mostrará que la justificación típica de las transferencias monetarias condicionadas es ilegítima en una comunidad política democrática. A través de la indagación de las razones por las que la carga de la prueba se coloca del lado de las transferencias incondicionadas, se mostrará que se apela a una noción controvertida de responsabilidad individual que cristaliza relaciones desiguales y que se basa en un diagnóstico empírico cuestionable.
\end{abstract}

Palabras clave: igualitarismo, protección social, legitimidad democrática, pobreza

\begin{abstract}
This paper analyzes the normative foundations of conditional cash transfers, a kind of poverty relief policy which has been massively implemented during recent years in Latin America. I intend to show that the conditional cash transfer's typical justification is illegitimate for a democratic political community. After reviewing the reasons why the burden of proof falls on unconditional transfers, I argue that the typical justification appeals to a controversial conception of individual responsibility that crystallizes unequal interpersonal relations and is based on a shaky empirical diagnosis.
\end{abstract}

Key words: egalitarianism, social protection, democratic legitimacy, poverty

Durante la última década, las transferencias monetarias condicionadas (TMC) se convirtieron en una herramienta de aplicación rápida y en gran escala que los Estados utilizan para alcanzar objetivos de política social como combatir la indigencia, aliviar la pobreza y romper con su transmisión intergeneracional. Los principios operativos de las TMC son simples: el Estado otorga dinero a las familias pobres con la condición de que adopten un comportamiento de inversión en capital humano, por lo común, hacer que los niños asistan a la escuela y a los centros de salud. Debido a su carácter masivo, algunas consecuencias de esta política son claras y poco controversiales: $30 \%$ de la población latinoamericana recibe de manera directa o indirecta alguno de estos 
beneficios; las inversiones en capital humano han aumentado de manera lenta pero regular y su grado de aceptabilidad social es relativamente alto en parte debido a la exigencia de condicionalidades.

A pesar de que el número de estudios sobre estas políticas sociales va en aumento, sólo una minoría de autores se ha ocupado con cierta profundidad del argumento normativo general que podría justificarlas. Entre ellos, es importante considerar los argumentos que Fiszbein y Schady ofrecieron y que se convirtieron en la principal herramienta teórica y programática del Banco Mundial, una de las instituciones más activas e influyentes en la promoción y defensa de estas políticas.

Uno de estos argumentos, el paternalista, parece ser el más importante. Su premisa básica es la miopía de los padres de las familias pobres: éstos se guiarán por las urgencias de lo cotidiano y mostrarán un grado considerable de desidia para realizar las inversiones necesarias para mejorar el futuro y las oportunidades de sus hijos. Así, los pobres necesitarán el "empuje (o el codazo) de los 'incentivos' del gobierno para comportarse en formas que sean 'buenas para ellos'. [...] Si se les permitiera a estos agentes actuar por cuenta propia, no serían capaces de elegir lo que más les conviene" (Fiszbein y Schady 2009, p. 53).

$\mathrm{El}$ argumento puede reconstruirse de la siguiente manera: si los pobres son responsables de su condición económica y ella puede dañar a terceros, entonces es legítimo adoptar políticas paternalistas que reemplacen su juicio defectuoso. Este argumento sería análogo a la interferencia legítima de un guardavidas de una playa que amonesta a un padre por jugar en forma imprudente con su hijo en un sector particularmente peligroso del mar.

En este artículo evalúo esta justificación y muestro que no podría aceptarse como legítima en una comunidad democrática. Para ello, no sólo tendré en cuenta los documentos de organismos internacionales y una selección de la bibliografía especializada en la evaluación del impacto de estas políticas, sino también la discusión entre las TMC y los proyectos incondicionados como la renta básica, el ingreso ciudadano e incluso el propio principio de la diferencia rawlsiano.

La estructura del texto es la siguiente: en la primera sección examinaré el concepto de responsabilidad que utilizan las TMC y analizaré si es empíricamente adecuado y si es normativamente válido; en la segunda sección mostraré que la justificación de estas políticas no es legítima en una comunidad democrática e igualitaria. 


\section{Padres desaprensivos y condicionalidades}

Las TMC diagnostican de manera explícita la existencia de una capacidad prudencial frágil en los progenitores pobres: simplemente no pueden considerar los beneficios a corto y largo plazo de invertir en el capital humano de sus hijos. Sin embargo, dado que los más dañados por estas decisiones son los niños, el diagnóstico implícito reviste una mayor gravedad moral que la mera imprudencia. Los primeros años de un individuo son fundamentales para su bienestar general; por lo tanto, el no asistir a los centros educativos ni a controles médicos periódicos tendrá una influencia muy negativa para el desarrollo de sus oportunidades reales. Este daño potencial constituye el núcleo de ese diagnóstico implícito según el cual los progenitores pobres son padres desaprensivos.

A modo de ilustración de este diagnóstico, supóngase que $A$ pasea por una playa y observa que el hijo de $B$ está ahogándose y que $B$ permanece impasible en la playa aunque podría ayudar a su hijo sin correr riesgos excesivos. Como $A$ no sabe nadar, promete a $B$ una cantidad de dinero para que éste rescate a su hijo. Así, $B$ es un padre desaprensivo al que no lo motivan los deberes morales hacia sus hijos. Este diagnóstico implícito exige que las TMC se comprendan como incentivos para cumplir con los deberes morales especiales hacia aquellos más cercanos a nosotros y que están temporalmente bajo nuestro cuidado. En este caso, lo que activa la interferencia estatal no es un juicio prudencial incorrecto, sino un carácter moral defectuoso. Este carácter moral defectuoso se revela con la consideración de que la forma más efectiva de lograr el cumplimiento de esos deberes morales no es impulsar el desarrollo de la capacidad moral de los progenitores, sino apelar a su capacidad para establecer planes racionales y estratégicos. En suma, las TMC consideran al progenitor pobre un inmoral que necesita incentivos económicos para cumplir con sus obligaciones.

Ahora bien, la justificación de esta interferencia estatal en las decisiones acerca de la inversión en capital humano depende de que exista una alta probabilidad de que los hogares pobres no cumplan con sus deberes morales hacia sus hijos a menos que se les impongan ciertas condicionalidades. Si esto fuera así, sería razonable desconfiar desde un inicio de que los hogares pobres fueran a cumplir con las obligaciones morales.

En los apartados siguientes se cuestionará la validez de esta justificación tomando en cuenta dos dimensiones. La primera es empírica y muestra algunos fenómenos típicos de las TMC que ponen en duda el 
diagnóstico de que los posibles beneficiarios son padres desaprensivos (1.1). La segunda dimensión muestra que la sospecha inicial hacia los beneficiarios de las TMC depende de una concepción inadecuada de la responsabilidad individual (1.2).

1.1. ¿Es empíricamente adecuado calificar de padres desaprensivos a los beneficiarios?

Imaginemos un padre que ni envía a sus hijos al colegio ni cuida su propia salud en forma periódica. Estas omisiones podrían deberse a dos tipos de razones, unas relativas a las oportunidades y otras a los juicios de valor que el sujeto realiza. Según las primeras, el padre quizá no tiene los recursos necesarios para enviarlos (el colegio queda muy lejos de su hogar, no tiene dinero para comprar los útiles y la vestimenta adecuada) o el costo de la oportunidad sería prohibitivo (no puede prescindir de los ingresos que genera el trabajo de los niños). De acuerdo con las segundas, el padre no hallaría razones de peso para cumplir con sus obligaciones morales porque, por ejemplo, no cree que el bienestar de sus hijos dependa de la salud ni de la educación o porque ni siquiera cree tener ese tipo de obligaciones.

Las TMC defienden el último tipo de razones a través del diagnóstico según el cual los posibles beneficiarios son padres desaprensivos. Si la causa del incumplimiento de las obligaciones morales no fuese una elección, entonces el Estado no tendría por qué condicionar la transferencia de recursos. ¿Existen argumentos empíricos o estadísticos que avalen esta elección o, por el contrario, se basa en una estigmatización de la conducta de los progenitores?

La respuesta empírica no es sencilla a pesar del número creciente de estudios sobre las TMC y del carácter masivo de las TMC en Latinoamérica. La evaluación de la información empírica y estadística es compleja y aún resulta discutible si pueden establecerse algunas regularidades concluyentes.

La incertidumbre respecto del diagnóstico no sólo se debe a la dependencia obvia del funcionamiento de las TMC de la situación económica más general de la región donde se aplican, sino a también a ciertos rasgos de diseño y de estructura. En primer lugar, excepto el caso atípico e icónico de las mexicanas Oportunidades y Progresa, las TMC no incluyen en su diseño instancias objetivas y externas de evaluación de impacto, o bien carecen de las capacidades burocráticas e institucionales para llevarla a cabo (Cecchini y Madariaga 2011, pp. 117-118). En segundo lugar, la comparación de las evaluaciones del impacto se 
dificulta por la utilización de metodologías diversas; se han empleado desde evaluaciones cuantitativas experimentales con grupos de control hasta simulaciones estadísticas, pasando por evaluaciones cualitativas que registran los procesos desencadenados por las TMC y encuestas de hogar (Maluccio, Adato y Skoufias 2010). En tercer lugar, es muy difícil distinguir entre el "efecto de la condicionalidad" y el "efecto del aumento de ingreso" (Cecchini y Madariaga 2011, pp. 117-118).

Estos obstáculos dificultan incluso el análisis de los efectos a corto plazo de las TMC que parecieran ser más visibles y regulares: el aumento de entre 4 y $8 \%$ de la asistencia y matriculación en las escuelas secundarias y primarias, la ausencia de mejoras significativas en el rendimiento académico, el aumento del gasto en alimentos y la disminución de la probabilidad de desnutrición infantil, el aumento del gasto en vestimenta, la reducción rápida de la indigencia y la escasa inserción en el mercado laboral formal (Pérez Ribas, Veras Soares y Hirata 2008, pp. 12-13; Draibe 2006; Gasparini y Cruces 2010). En relación con cada uno de estos datos tiene sentido preguntarse por el verdadero alcance causal del "efecto de la condicionalidad", es decir, hasta qué punto el motor del cambio en la conducta es el ingreso obtenido o la condicionalidad impuesta.

Como ejemplo de esta incertidumbre es relevante considerar tres fenómenos comunes. El primero surge de un análisis cuantitativo sencillo: comparar los porcentajes de vacunados y escolarizados antes y después de la transferencia monetaria condicionada. En Argentina, durante el periodo entre 2007 y 2011 se mantuvo a $1.4 \%$ de los niños de los centros urbanos sin asistir a colegios primarios y a $9 \%$ de los adolescentes sin asistir a escuelas secundarias (González y Tuñón 2012). ${ }^{1}$ La transferencia monetaria condicionada Chile Solidario/Ingreso Ético Familiar aumentó la matrícula escolar básica entre 7 y 9 puntos porcentuales, y las visitas de prevención sanitaria de niños menores de seis años en zonas rurales entre 4 y 6 puntos porcentuales (Vargas 2011, p. 33). Ahora bien, el papel causal de estas TMC se debilita cuando se considera que ambas variables ya eran superiores a $85 \%$ antes de la aplicación de la transferencia monetaria condicionada (Huneeus y Repetto 2013, p. 7) o que la matrícula ya aumentaba entre 0.8 y $2 \%$ anual desde 2006 (Huneeus y Repetto 2013, p. 230). Aun de forma provisional y sin perder de vista ciertas regularidades, ${ }^{2}$ estos datos permiten sugerir que, de una u otra forma, los padres pobres ya cumplían

${ }^{1}$ La transferencia monetaria condicionada argentina se comenzó a aplicar en 2009. Estos datos se confirman en el Censo Nacional de 2010.

${ }^{2}$ Por ejemplo, Cechinni y Madariaga 2011 (p. 118) establecieron que los incre- 
con las obligaciones hacia sus hijos al margen de los efectos del "ingreso" y la "condicionalidad".

Un segundo fenómeno consiste en el efecto "par", identificable en las TMC Progresa, Tekoporá y otras. Este efecto puede definirse como un tipo de externalidad positiva según la cual los hogares excluidos del beneficio mejoran, no obstante, su bienestar ya sea porque la economía local mejora o porque estos hogares responden a la interacción con hogares beneficiados (Veras Soares, Pérez Ribas y Hirata 2008, p. 13). Por ejemplo, en los lugares donde se puso en marcha Progresa, la matrícula escolar de los niños que no participaban en el programa aumentó $5 \%$ en comparación con las localidades en las que no se había aplicado esa transferencia monetaria (Bobonis y Finan 2009).

El último fenómeno surge de casos en los que las condicionalidades fueron leves porque su cumplimiento no se monitoreó institucionalmente debido a limitaciones estatales de control. La transferencia monetaria ecuatoriana Bono de Desarrollo Humano se anunció como una que exigía la matriculación de los niños en la escuela primaria y secundaria aunque, en realidad, nunca se aplicaron controles ni se ligó el cobro al cumplimiento de la condicionalidad. En un trabajo muy interesante, Schady y Araujo 2008 aprovecharon esta anomalía para comparar de manera no experimental las tasas de matriculación escolar según si el hogar creía o no que había condicionalidades. El resultado fue que la matriculación aumentaba de 6 a $8 \%$ si los padres creían que había condicionalidades adjuntas. Otro dato similar proveen De Brauw y Hoddinott 2011 respecto de Progresa; esta transferenica monetaria condicionada entregaba a los padres beneficiarios un formulario para verificar los porcentajes de asistencia (el Formato E1), que debían firmar las autoridades educativas. En algunas localidades rurales los padres recibieron la transferencia pero no el formulario, de forma que la condicionalidad no se controló. A través de una comparación entre distintos grupos de beneficiarios de estas regiones, los autores mostraron que $88.4 \%$ de los hogares que recibieron el beneficio y el formulario cumplió las condicionalidades y que quienes no habían recibido el formulario pero sí el beneficio cumplieron las condicionalidades en $85.2 \%$ de los casos. Al mismo tiempo, los autores averiguaron si los beneficiarios sabían o no que la transferencia tenía por condición la matriculación. La diferencia resultó mayor: la matriculación era de $89.2 \%$ en

mentos en la matrícula tienden a ser menores en regiones donde los niveles de referencia son más altos y en las etapas con mayor asistencia. Por ejemplo, la TMC colombiana Familias en Acción registra un aumento mayor de asistencia a la escuela secundaria que a la primaria, en la que la asistencia ya era muy elevada.

Diánoia, vol. LXII, no. 79 (noviembre de 2017). 
quienes sabían de la condicionalidad y recibieron el formulario mientras que descendió a $82.2 \%$ en quienes ni recibieron el formulario ni sabían de la condicionalidad.

Estos fenómenos no son suficientes para negar que el efecto "condicionalidad" marca una diferencia en el cumplimiento de las obligaciones parentales de cuidado. Como queda claro, la probabilidad de cumplimiento aumenta en forma débil en la medida en que la condicionalidad está presente y es clara. Sin embargo, también presentan una contracara que merece examinarse. Si el efecto "condicionalidad" tuviese un papel causal tan importante, ¿no debería ser mayor la diferencia entre quienes saben que el beneficio es condicional y quienes no lo saben?, ¿no debería ser relevante que más de $80 \%$ de aquellos que no sabían de las condicionalidades las cumplieran de todos modos? En todo caso, la evidencia a favor del poder causal de las condicionalidades dista de ser concluyente.

Sin lugar a dudas, y como aceptan tanto los defensores de las condicionalidades como sus detractores, hace falta una mayor investigación estadística y mejores evaluaciones de impacto para extraer conclusiones significativas y con peso probatorio. Sin embargo, estos tres fenómenos sugieren que se sobrestiman la importancia y la eficacia de las condicionalidades. En otras palabras, la carga de la prueba se coloca de manera arbitraria del lado de las transferencias incondicionales, en especial si se tiene en cuenta que en las regiones donde las TMC son de aplicación masiva existe una larga trama de inacción estatal, desigualdad y ausencia de establecimientos educativos y sanitarios de calidad aceptable. Si retomamos el ejemplo inicial de esta sección, se puede ver que las TMC deslindan completamente de mayores responsabilidades al Estado o a la comunidad política; es como si el Estado tuviera la capacidad de contratar guardavidas para cuidar la playa y no lo hiciera, no diseñara carteles más llamativos, permitiera que el niño ingresara sin supervisión en aguas embravecidas y sólo cuando estuviera ahogándose ofreciera el incentivo.

En el próximo apartado exploraré las consecuencias normativas de esta asignación de la carga de la prueba del lado de las transferencias incondicionales y mostraré que depende de una concepción como mínimo controvertida de la responsabilidad individual.

\section{2. ¿Qué significa que los pobres sean responsables de su pobreza?}

En el apartado previo establecí que existe un margen para dudar razonablemente del diagnóstico inicial que califica a los posibles beneficiarios de padres desaprensivos y que, por lo tanto, no está claro por qué 
la carga de la prueba debe atribuirse al defensor de las transferencias incondicionadas. En otros términos, si la desconfianza inicial hacia los beneficiarios de las TMC no se justifica con los datos estadísticos o empíricos, ¿cómo podría justificarse? Aquí analizaré y rechazaré una razón que podría cumplir esta función y que se vincula con una concepción de la responsabilidad individual.

Las TMC están impregnadas de creencias y percepciones públicas paradójicas. Por ejemplo, sus detractores conservadores sospechan que los beneficiarios son vagos e irresponsables y que el ingreso que reciben lo utilizan para financiar sus vicios y decisiones imprudentes. Por el contrario, los defensores de las TMC califican lo anterior de prejuicios infundados, pero aplican condicionalidades fuertes, es decir, ligan el beneficio a su cumplimiento y control. ${ }^{3}$

La discusión entre estas posiciones es sólo aparente y está condenada al fracaso porque su resolución exige aceptar una posición metafísica necesariamente controvertida respecto de la discusión clásica entre el libre albedrío y el determinismo. A pesar de la aparente antinomia, ambos enfoques defienden la tesis de que la responsabilidad de los pobres por su propia situación determina la extensión de la obligación del Estado; mientras que los detractores afirman que los pobres son en efecto responsables de su situación y que, por lo tanto, deberían renunciar a los reclamos para mejorarla, los defensores niegan que los pobres sean en realidad responsables de su situación y, justo por eso, concluyen que el Estado está obligado a mejorar su situación. ${ }^{4}$

La tesis común confunde dos sentidos distintos de responsabilidad que Thomas Scanlon distinguió analíticamente. El primer sentido, el de la responsabilidad atributiva, se utiliza cuando se afirma que la acción $A$ es propia del sujeto $B$ en virtud de algún hecho de la relación entre la acción y $B$; dado ese hecho, el agente puede ser reprobado o alabado por su acción. El segundo sentido, el de la responsabilidad sustantiva, surge cuando se afirma que el sujeto $B$ debe tanto hacerse cargo de las consecuencias de la acción $A$ (Scanlon 1998, pp. 248-249) como liberar a los demás de las obligaciones de asumir esas consecuencias.

\footnotetext{
${ }^{3}$ Ernesto Sanz, senador de un partido político socialdemócrata de Argentina, afirmó que la Asignación Universal por Hijo "se está yendo por la canaleta de la droga y el juego". A su vez, el ex ministro de educación argentino, Alberto Sileoni, afirmó que "nosotros confiamos en los beneficiarios, confiamos en la gente y el buen uso que hace".

${ }^{4}$ Scanlon 2008, p. 199 atribuye esta misma tesis, común a las posiciones de izquierda y conservadoras en el discurso político.
} 
La idea central de la distinción consiste en separar las obligaciones de la justicia hacia los otros de las reacciones pertinentes de reproche o elogio que podemos tener hacia un agente. De acuerdo con Scanlon, la conclusión de que $B$ es sustantivamente responsable de $A$ se deriva, por un lado, de premisas que definen cuándo una distribución de cargas y beneficios es equitativa (Scheffler 2005, p. 9) y, por otro lado, de qué desigualdades son compatibles con un sistema equitativo de cooperación. Así, este sentido de responsabilidad surge del establecimiento de obligaciones de justicia. Por el contrario, la responsabilidad atributiva depende de qué factores o condiciones puedan excusar a un agente por su acción y hacerlo o no merecedor de ciertas reacciones morales como el perdón, el elogio o el reproche. Así, la atribución de este sentido de responsabilidad es adecuada si conocemos qué constituye una acción genuinamente voluntaria.

Como está claro, la tesis compartida extrae consecuencias para la responsabilidad sustantiva a partir de la responsabilidad atributiva (Queralt 2015; Blake y Risse 2008, pp. 178-181). Dicho de otra forma, hace depender las obligaciones de la comunidad política del hecho de si un individuo es en efecto un agente; si el individuo es censurado por su comportamiento, debe asumir las consecuencias negativas producidas. La teoría scanloniana ha ofrecido dos razones para pensar que esta inferencia es incorrecta.

La primera razón reside en que las condiciones para asignar responsabilidad atributiva y responsabilidad sustantiva son fundamentalmente divergentes. De acuerdo con Scanlon, asignar responsabilidad atributiva no requiere que el agente tenga un control total sobre todos los factores que operan causalmente en sus deliberaciones y decisiones ni que haya podido evitar esos factores (Scanlon 2008, pp. 193-199) pero sí que exista una conexión estable entre la capacidad para evaluar razones y la acción o actitud del agente (Scanlon 1998, p. 221). ${ }^{5}$ Por ejemplo, un individuo que no ahorra dinero para tiempos de crisis sigue siendo responsable atributivamente y es objeto de censura moral incluso si no eligió su actitud general de poca aversión al riesgo, si no pudo evitar que las razones del bienestar inmediato oscurecieran por

${ }^{5}$ En este sentido, Scanlon adopta una posición compatibilista de acuerdo con la cual la responsabilidad moral tendría lugar incluso en un mundo determinista (Matravers 2003, pp. 69-70). Como él mismo señala, esta posición es similar a la de Harry Frankfurt (Scanlon 1998, pp. 291-293). Para una introducción detallada sobre cómo concepciones distintas de responsabilidad atributiva justificarían parámetros distintos de justicia distributiva, véase Hurley 2003, pp. 138-149 y Queralt 2014, pp. 130-147. 
completo sus deliberaciones sobre su bienestar futuro y si sus figuras paternas castigaban físicamente la prudencia económica.

En cambio, la asignación de responsabilidad sustantiva sí requiere que el agente tenga la oportunidad de elegir y, por lo tanto, una concepción de cuándo esas oportunidades son "adecuadas" (Scanlon 1998, p. 286), es decir, de cuándo hubiera sido razonable esperar que el agente eligiera de otra forma (Voorhoeve 2008). Así, un agente debe hacerse cargo de su situación y renunciar a pedir la asistencia de los otros si tuvo las oportunidades adecuadas para evitar la acción o la situación. Por ejemplo, el imprudente con el dinero puede pedir de manera legítima asistencia si no se le enseñó el valor de la prudencia económica o si, dada su posición económica, el costo de la oportunidad de ahorrar era demasiado elevado. De esta forma, un mismo individuo podría ser responsable atributivamente de la acción $B$ pero no responsable sustantivamente de la acción $B$.

La segunda razón para rechazar la inferencia de responsabilidad sustantiva a partir de la responsabilidad atributiva surge del principio de que hay que procurar que la justicia distributiva sea "metafísicamente mínima" (Matravers 2003, p. 75; Long 2016), es decir, impedir que los inevitables desacuerdos sobre la justicia pública se solucionen con posiciones metafísicas controvertidas que no todos los ciudadanos razonables considerarían aceptables. En otros términos, que el imprudente en el terreno económico tenga derecho a la asistencia de los demás no depende de que alguna concepción de la responsabilidad atributiva lo señale como el autor "genuino" de sus decisiones, sino de un razonamiento normativo según el cual tal asistencia sea una obligación que le debe su comunidad política.

Con todo, esta distinción es por sí sola insuficiente para rechazar la tesis compartida. Los defensores podrían afirmar: "Nosotros no confundimos estos dos sentidos de responsabilidad, sino que creemos que hay una implicación entre uno y otro. Quizá no seamos tan prolijos, pero entendemos que son conceptos distintos." Esta respuesta es correcta. Las TMC no confunden estos dos sentidos porque, de hecho, afirman que la responsabilidad atributiva de los pobres no implica de manera directa que ellos deban hacerse cargo de todas las consecuencias de sus decisiones; por el contrario, las TMC otorgan el beneficio a pesar de la responsabilidad atributiva.

Sin embargo, las TMC sí conllevan una implicación débil: la responsabilidad atributiva determina cómo la comunidad política debe tratar al individuo. Dado que hay que censurar al individuo por su comportamiento, su comunidad política está autorizada a interferir para reem-

Diánoia, vol. LXII, no. 79 (noviembre de 2017). 
plazar su juicio sobre la inversión en capital humano. Hay dos razones para rechazar esta implicación débil. La primera es que las TMC no surgen como respuesta a contextos de amplia igualdad de oportunidades o donde la comunidad política ha cumplido bien con sus obligaciones hacia los ciudadanos. Dado que el criterio determinante para la pertenencia al grupo beneficiario es la responsabilidad atributiva y dado que ésta no requiere tener opciones adecuadas, la obligación estatal no es mejorar las oportunidades de los individuos, sino dar "codazos" para que los pobres modifiquen sus actitudes hacia la inversión en capital humano. Dicho de una manera más cruda, la obligación del Estado no es construir más hospitales en lugares remotos, sino ofrecer dinero a cambio de que los pobres se desplacen hasta los hospitales lejanos. Así, la comunidad política adopta posiciones extremadamente autocomplacientes y poco demandantes.

En segundo lugar, la implicación débil introduce una desigualdad de trato injusta entre los progenitores de bajos recursos al distinguir entre pobres merecedores y no merecedores. Los excluidos de las TMC serían quienes no permiten que el mejor juicio del Estado se imponga sobre sus elecciones, es decir, el filtro de las condicionalidades permite confirmar la sospecha de que los potenciales beneficiarios hacen de sus elecciones incorrectas un componente esencial e imposible de erradicar de su identidad. Ahora bien, los estudios empíricos confirman que los excluidos resultan ser los más pobres de los pobres, es decir, los que tienen menor información, menor acceso a los servicios y funcionarios públicos y, por lo tanto, menores oportunidades (Kerstenetzky 2009, p. 65). ${ }^{6}$ Así, la comunidad política y el Estado tratan de manera más severa a los más pobres de los pobres, es decir, a quienes han tenido menos oportunidades, por considerarlos reacios a modificar sus creencias y actitudes hacia la inversión en capital humano.

De esta manera, las TMC se basan en una concepción de la responsabilidad individual, la responsabilidad atributiva, que limita las obligaciones de la comunidad política hacia los sectores menos aventajados al mismo tiempo que introduce una distinción inequitativa entre pobres merecedores y pobres no merecedores.

\footnotetext{
${ }^{6}$ Otro ejemplo es el requisito de la Asignación Universal por Hijo, de acuerdo con el cual no podían ser beneficiarios los alumnos que iban a escuelas privadas. Debido a numerosas críticas, presiones sociales y a que en 2009 el $33 \%$ de los alumnos del segundo quintil de ingresos asistía a escuelas privadas, se dio marcha atrás con la suspensión de 42 mil beneficios por estas razones (Pautassi, Arcidiácono y Straschnoy 2013, p. 27).
} 
2. Paternalismo, juicios de responsabilidad y legitimidad democrática

La sección anterior mostró que es al menos razonable cuestionar la validez empírica y normativa del diagnóstico que califica de padres desaprensivos a los beneficiarios y que constituye la base teórica de las TMC. Por ello es preciso profundizar en la pregunta de por qué la carga de la prueba se coloca del lado de las transferencias monetarias incondicionales y no de las TMC.

En esta sección ofreceré esa profundización, la cual será útil para mostrar que la justificación de las TMC es ilegítima en una comunidad política de iguales. En el primer apartado, mostraré que las TMC no constituyen un caso de paternalismo inaceptable o impermisible ya que lo que intentan proteger no es, como suele suponerse, a individuos imprudentes, sino a terceros que resultarían dañados por decisiones imprudentes (2.1). En el segundo apartado defenderé que, a pesar de ello, las TMC no son legítimas desde un punto de vista democrático porque colaboran a perpetuar relaciones desiguales entre los ciudadanos de una comunidad política (2.2).

\section{1. ¿Son paternalistas las TMC?}

Como ya se mencionó, tanto sus defensores como sus críticos consideran las TMC como casos de paternalismo estatal (Freeland 2007, p. 75; Lo Vuolo 2011, p. 211; Pautassi, Arcidiácono y Royo 2010, p. 240; Fiszbein y Schady 2009, p. 53). Por lo tanto, su justificación debería apelar y mostrar la incapacidad de los padres de hogares pobres para considerar en forma adecuada las consecuencias negativas de sus acciones.

Antes de analizar si una justificación así es adecuada, son necesarias dos aclaraciones previas, una conceptual y otra normativa. En primer lugar, no hay un consenso respecto de la definición filosófica de paternalismo y, por lo tanto, la caracterización de las TMC como paternalistas depende de qué se incluya en el ámbito del control legítimo del agente que sufre una interferencia. Por ejemplo, las TMC no serían paternalistas si se adopatara la definición clásica de Gerald Dworkin, según la cual $A$ actúa en forma paternalista respecto de $B$ al realizar (u omitir) la acción $Z$ si y sólo si: i) $Z$ interfiere con la autonomía de $B$, ii) $A$ la realiza sin el consentimiento de $B$, y iii) la razón para $Z$ es que mejorará el bienestar o promoverá los intereses de $B$ (Dworkin 2013, p. 29). En las TMC la condición iii) no aparece porque la interferencia se justifica por el bienestar de un tercero (el hijo de $B$ ). Ahora bien, según la definición de Shiffrin 2000 (p. 218), las TMC serían prima facie paternalistas. En ésta, la condición iii) se reemplaza con iii $_{1}$ ): la acción $Z$ se orienta a 
los propios intereses de $B$ o a los asuntos que caen legítimamente en el ámbito del control de $B$. Puesto que es razonable suponer que las decisiones en torno al cuidado de los hijos caen en el control legítimo de $B$, las TMC serían acciones paternalistas.

La segunda aclaración se relaciona con las implicaciones normativas de la caracterización. El hecho de que una política se etiquete o no como paternalista no resuelve ninguna disputa moral sustantiva ya que, por ejemplo, no es raro hallar políticas estatales paternalistas aceptables, como la obligación de utilizar cinturones de seguridad o la prohibición de nadar en las playas de aguas embravecidas. En todo caso, la justificación de las TMC debería mostrar que el tipo de interferencia paternalista es aceptable o permisible.

¿Constituyen entonces las TMC un caso de "paternalismo" aceptable? Considérese el ejemplo de la prohibición estatal de nadar en una playa embravecida. Como el Estado prevé que hay individuos que no razonan con prudencia sobre las consecuencias y los peligros de nadar allí, interfiere en sus opciones disponibles con el fin de proteger su bienestar.

¿Son las TMC comparables con este caso típico? La apelación a la falta de prudencia de los progenitores pobres conduciría a una respuesta positiva. Sin embargo, la analogía es demasiado débil para sostenerse porque el objetivo de las TMC no es el bienestar del propio progenitor imprudente, sino el de un tercero, el del niño que ve disminuidas sus oportunidades por esa capacidad defectuosa para juzgar sobre lo que es prudente.

Ahora bien, incluso si se aceptara la definición de Shiffrin y estas interferencias fueran paternalistas, la pregunta moral se mantendría: ¿serían aceptables? La respuesta es positiva por cuatro razones.

En primer lugar, el daño a la autonomía futura de un tercero sin la intervención paternalista es difícil de exagerar. Si bien antes cuestioné la magnitud de esta probabilidad, está claro que el daño que se produce en un niño por no asistir al colegio y por no cuidar su salud difícilmente puede sobredimensionarse. ${ }^{7}$

${ }^{7}$ La reciente discusión en Estados Unidos sobre la obligatoriedad de las vacunas y la libertad parental para decidir cómo proteger al niño a través de medidas alternativas podría cuestionar esta razón. Si bien es importante notar que esa discusión se originó por una serie de creencias con poco respaldo científico — por ejemplo, que la vacunación producía autismo o que contenía conservantes tóxicos-, la razón principal para diluir la objeción de paternalismo indebido es que el Estado no podía desentenderse totalmente de su obligación de proteger la autonomía futura de individuos actualmente vulnerables y que los beneficios de la vacunación obli- 
En segundo lugar, las condicionalidades no se vinculan con una educación religiosa o con cuidados médicos que favorezcan determinados roles o profesiones, sino con un conjunto mínimo de deberes de protección hacia los futuros ciudadanos. Así, la interferencia estatal deja un espacio relativamente amplio de opciones para educar a los niños, sin imponer un ideal de vida buena.

En tercer lugar, las condicionalidades no imponen cargas tan onerosas y restrictivas para los progenitores como las de, por ejemplo, justificar cada uno de los gastos o meramente ofrecer cupones para consumos específicos.

En cuarto lugar, es muy difícil afirmar que cumplir o no con los deberes morales forma parte de las opciones que constituyen la autonomía humana. Si incentivar la reducción del incumplimiento de estas obligaciones es inaceptablemente paternalista, también lo sería alabar a nuestros amigos por cumplir sus promesas.

De esta forma, las TMC o bien no son paternalistas o bien no son inaceptablemente paternalistas, según la definición que se adopte. En cualquier caso, la defensa y promoción de la autonomía futura de los más vulnerables es el elemento que carga con mayor peso normativo en la justificación de estas políticas masivas de alivio de la pobreza.

Así, si las TMC no son inaceptablemente paternalistas, ¿de dónde emana su legitimidad democrática? La respuesta más simple es que surge de la combinación del diagnóstico que califica a los beneficiarios de padres desaprensivos con el daño futuro a la autonomía de los menores. Tal combinación legitimaría la relación de desconfianza entre la comunidad política y el sujeto que sufre la interferencia y, entonces, sería razonable condicionar los beneficios. En el siguiente apartado mostraré que tal combinación es insuficiente para conferir legitimidad a este tipo de interferencias estatales.

\subsection{Legitimidad democrática, relaciones desiguales y desigualdad de trato}

Una de las obligaciones fundamentales y más demandantes del Estado es la de proteger a los sujetos vulnerables que no pueden decidir por sí solos el destino de su vida. Como se consideró en el apartado anterior, las interferencias de las TMC se justifican por ser una forma relativamente eficaz de cumplir con esta obligación. Ahora bien, ¿significa esto

gatoria superan con creces sus riesgos. Para un análisis muy interesante y detallado del caso, véase Pierik 2015.

Diánoia, vol. LXII, no. 79 (noviembre de 2017). 
que ese objetivo las convierte en políticas públicas completamente legítimas?, ¿implica que la carga de la prueba debe colocarse del lado de las transferencias monetarias incondicionadas? A continuación responderé negativamente a ambas preguntas.

El principal recurso teórico de los defensores de las TMC consiste justo en la apelación a la legitimidad democrática. De acuerdo con el texto fundamental de Fiszbein y Schady 2009, la carga de la prueba está situada adecuadamente por una razón de economía política según la cual las condicionalidades contribuyen a la legitimidad y aceptabilidad general del subsidio otorgado. Las condicionalidades típicas (enviar los niños al colegio y vacunarlos) se consideran signos de un "buen comportamiento" de los padres pobres y, por lo tanto, "Algunos votantes que objetan las 'donaciones' incondicionales pueden ser menos adversos a 'recompensar' a pobres 'meritorios' que invierten en la educación o salud de sus hijos" (Fiszbein y Schady 2009, p. 64). El argumento podría reconstruirse de la siguiente forma:

1) Excepto la TMC nicaragüense Red de Protección Social (Cecchini y Madariaga 2011, p. 157), todas las TMC de la región se financian con fondos públicos.

2) La legitimidad democrática exige que las políticas públicas se justifiquen con razones y criterios ampliamente compartidos.

3) Tanto los beneficiarios de las TMC como los ciudadanos que las financian coinciden en utilizar criterios de responsabilidad atributiva para distribuir cargas y beneficios (Cruces, Rovner y Schijman 2007, p. 45; Kerstenetzky 2009). ${ }^{8}$

Así, las políticas incondicionales serían ilegítimas porque no contarían con el apoyo de los ciudadanos en general ya que no podrían vincularla con razones que ellos aceptan y reconocen. Tales políticas serían totalmente inestables y quedarían expuestas a las denuncias demagógicas

\footnotetext{
${ }^{8}$ Según una encuesta realizada en 2007 por el Centro de Estudios Distributivos, Laborales y Sociales de Argentina, la importancia de las condicionalidades orientadas a la acumulación de capital humano no puede soslayarse: $51 \%$ de los entrevistados mencionó que, para acceder al subsidio, debía controlarse la escolaridad de los niños. Si bien la encuesta es previa a la instauración de la Asignación Universal por Hijo y se refería a los planes sociales previos, no es aventurado extrapolar sus conclusiones a este programa en cuestión. Una de sus conclusiones relevantes es que una gran mayoría de los entrevistados (83\%) opinó que, a cambio de recibir un plan, la contraprestación debería relacionase con el trabajo, y cerca de $80 \%$ que debería relacionarse con la educación.
} 
de los beneficiarios como "vagos", "irresponsables"; los propios programas serían calificados de "financiadores de una cultura de dependencia del estado" o, peor aún, de crear sujetos clientelares dependientes de los partidos políticos gobernantes (Pinzani 2014, pp. 158-162). Así, las consideraciones de responsabilidad individual que implican las TMC deben incluirse por razones de legitimidad ya que, como señala Kymlicka, en su ausencia:

los ciudadanos podrían comenzar a percibir que los criterios de impuestos y transferencias del Estado de Bienestar son, en el mejor caso, una recompensa a cualquier interés especial que esté en el poder y, en el peor escenario, un modo moralmente perverso de castigar a los contribuyentes cumplidos para que subsidien a los imprudentes o a los perezosos. (Kymlicka 2006, p. 30)

Sin embargo, el razonamiento es ambiguo. El mero hecho de que una idea esté presente en el discurso político de una sociedad y que los ciudadanos la utilicen para discutir asuntos públicos no significa que deba ser la razón que triunfe siempre sobre otras ideas. En este sentido, el argumento corre el riesgo de confundir una cuestión de legitimidad con una de pragmatismo político, es decir, de creer que las consideraciones razonables son idénticas a las razones que producen contextualmente un acuerdo estratégico entre visiones rivales.

De acuerdo con la metodología política rawlsiana, para que un juicio - como que la justicia distributiva sea sensible a consideraciones de responsabilidad atributiva - sea parte de la razón pública - y, por lo tanto, su uso sea legítimo- debe ponerse en equilibrio reflexivo con otros principios y otros juicios (Rawls 1999, pp. 40-45). Tal equilibrio reflexivo es el resultado de un proceso deliberativo intersubjetivo en el que las leyes y el poder político se justifican públicamente sobre la base de razones mutuamente aceptables (Rawls 2004, pp. 51-58). De esta forma, la legitimidad de un criterio de responsabilidad individual para asignar o rechazar beneficios sociales depende no de que muchos ciudadanos lo utilicen con frecuencia, sino de que puedan justificarlo públicamente.

A continuación defenderé tres razones para sostener que este criterio de responsabilidad no superaría una prueba interesante de legitimidad.

La primera de ellas es que este criterio entra en conflicto con las razones por las que el Estado debe hacerse cargo de combatir la transmisión intergeneracional de la pobreza. El Estado tiene esta obligación porque son sus ciudadanos quienes sufren sus consecuencias y no por

Diánoia, vol. LXII, no. 79 (noviembre de 2017). 
la mera existencia del fenómeno. En otras palabras, las razones para combatir la transmisión de la pobreza se activan sólo cuando el tipo de relación entre los más aventajados y los menos aventajados no es meramente causal, sino que ya está, de alguna manera, moralizada. La comunidad política organizada alrededor de un régimen democrático plantea un tipo especial de relaciones moralizadas, una relación igualitaria entre los ciudadanos. Estas relaciones, sean en el ámbito económico, social o político, se regulan por un principio normativo, el del estatus igualitario. Según este principio, la igualdad de estatus de cada ciudadano es independiente de las circunstancias que los diferencian como individuos únicos y de la prudencia o imprudencia de sus elecciones (Anderson 1999, pp. 312-315; Scheffler 2003, pp. 21-24; Fourie 2012). Así, la ciudadanía no está delimitada por ningún criterio de responsabilidad atributiva. El empleo de ese criterio constituiría un tipo de relaciones desiguales entre los propios ciudadanos, una especie de ciudadanía de segunda clase. Los más aventajados estarían legitimados a extraer consecuencias redistributivas a partir de los juicios de responsabilidad atributiva que ellos mismos realizan sobre la conducta de los menos aventajados, mientras que éstos no estarían legítimamente habilitados para hacerlo. De esta forma, la utilización de este criterio de responsabilidad para distribuir beneficios entra en conflicto con el ideal de ciudadanía democrática.

La segunda razón parte de las conclusiones que surgieron en el análisis de la distinción de Scanlon entre responsabilidad atributiva y sustantiva y apela al criterio rawlsiano de legitimidad en sociedades que aceptan el pluralismo razonable. El empleo de un criterio de responsabilidad individual para asignar beneficios de protección social implica tomar posiciones metafísicas necesariamente discutibles acerca de qué condiciones y características debe tener una decisión para ser propia del agente y acerca de cuán sensible debe ser el razonamiento del agente a las razones moralmente "correctas" (Queralt 2015 y Scheffler 2005). La fijación de estas posiciones en documentos legales y en reglas públicas y concretas de aplicación amenazaría la posibilidad de llegar a un consenso superpuesto en contextos de pluralismo razonable porque los ciudadanos podrían rechazarlas razonablemente y considerar que el Estado es parcial respecto de algunas teorías metafísicas controvertidas, como las relacionadas con la identidad y la libertad (Rawls 1993, pp. 134-150 y Long 2016, pp. 110-114). Por el contrario, el compromiso con la legitimidad rawlsiana exige que los ciudadanos renuncien a la imposición de posiciones metafísicas y doctrinas comprehensivas como justificación del poder coercitivo porque entienden que eso equivaldría 
a que otros individuos (también razonables) se vean forzados a vivir bajo reglas que, de acuerdo con sus doctrinas del bien, consideran falsas (Rawls 1993, pp. 217). Así, un sistema de protección social como elemento de una concepción política de la justicia sólo se justificaría si se convierte en el foco de un consenso entrecruzado entre distintas doctrinas comprehensivas razonables, esto es, si se presenta desde un punto de vista independiente que no exige la adopción de una doctrina comprehensiva particular para su aceptación y justificación (Rawls 1993, pp. 8-14). De esta manera, la utilización de un criterio de responsabilidad atributiva para asignar beneficios de protección social conlleva la siguiente paradoja: si por razones de legitimidad se incluyen creencias populares acerca de la libertad, la responsabilidad y el merecimiento, se obtiene un criterio ilegítimo.

La tercera razón para cuestionar la legitimidad de este criterio es de orden procedimental. Un proceso de justificación intersubjetivo brinda un grado máximo de legitimidad cuando se realiza en condiciones de igualdad relativa; cuando éstas están presentes, no se excluyen de forma arbitraria posiciones, el poder económico no se traduce en poder político, se minimizan los poderes de la amenaza, las razones se dirigen a iguales que tienen las mismas capacidades y oportunidades para cuestionar, modificar e incluir nuevas perspectivas e informaciones, etc. (Bohman 1996). Como ya se señaló, el contexto histórico de las TMC latinoamericanas no cumple con muchas de estas condiciones: hay una profunda desigualdad histórica, la vulnerabilidad de los peor situados se incrementa por el carácter crónico de los problemas de desempleo y trabajo informal, existen altos niveles de pobreza e indigencia y las oportunidades educativas, sanitarias y relacionales distan de ser igualitarias.

En este tipo de contextos desiguales, la aceptación de las condicionalidades por parte de los beneficiarios y de los contribuyentes no sólo debe relativizarse, sino que puede derivarse de la propia injusticia de tales condiciones: ante la alternativa de continuar viviendo en una trama de inacción estatal y de exclusión completa de las instituciones de protección social, la aceptación de condicionalidades severas y desiguales se presenta como una estrategia completamente racional. Por ejemplo, si a un individuo desempleado por mucho tiempo se le ofrece un salario bajo y con pocas garantías, es probable que lo acepte. Sin embargo, es difícil que tal aceptación pueda contar como prueba de la legitimidad de la práctica.

Por otro lado, esta aceptación no sólo se deriva de un tipo de relaciones desiguales, sino que colabora a perpetuarlas. La relación entre 
el beneficiario de una TMC y su comunidad política es de sospecha y desconfianza; se exige la condicionalidad porque se sospecha que los beneficiarios no invertirían en capital humano con un mero aumento de su ingreso. Así, los funcionarios públicos y los ciudadanos se justifican al mostrarse suspicaces y desconfiados hacia los potenciales beneficiarios, incluso si éstos llevan años cumpliendo con las condicionalidades (Wolff 1998, pp. 108-109). En una relación de iguales, la desconfianza inicial puede ser razonable y no violar la simetría de la relación. Por ejemplo, un individuo está justificado en desconfiar de un amigo que acaba de salir de un proceso de desintoxicación de drogas; sería razonable que no le preste una suma considerable de dinero, que no deje sus hijos a su cuidado y, en general, que no confíe en que pueda superar circunstancias muy estresantes. Sin embargo, si luego de un periodo en el que el amigo no ha recaído en su adicción el grado de desconfianza se mantiene incólume, la relación igualitaria necesariamente deja de serlo. Uno de los participantes no está dispuesto a dejar de realizar juicios acerca de la conducta y responsabilidad del otro, mientras que éste genera hábitos y conductas estratégicas para complacer e internalizar los juicios de desconfianza del otro.

Los defensores de las TMC podrían dudar de la fuerza de esta última razón. Después de todo, estas políticas estipulan un umbral encima del cual los juicios de desconfianza dejan de justificar las obligaciones de probar el cumplimiento de los deberes morales por parte de los beneficiarios. Este umbral es el empleo formal, en el cual los beneficiarios de las asignaciones familiares ya no tienen que demostrar en forma periódica el cumplimiento de sus obligaciones; se da por supuesto que la disponibilidad segura y estable de un ingreso promedio es garantía de este cumplimiento, sin necesidad de demostrar otras condicionalidades. Sin embargo, la satisfacción de este umbral parece ser sólo teórica; como muestra la mayoría de las evaluaciones de las TMC, la probabilidad de las "opciones de salida" del sistema condicional es prácticamente desechable (Cecchini y Martínez 2011, pp. 189-191) Así, las TMC promueven relaciones desiguales y asimétricas que se perpetúan a lo largo del tiempo y que estimulan la creación y estabilidad de estrategias racionales de anticipación y manipulación de conductas.

Las tres razones por las cuales las TMC, y en especial las que estipulan condicionalidades fuertes, son ilegítimas, muestran un límite importante que el razonamiento moral y práctico debe afrontar. En contextos de profunda desigualdad y de pobreza crónica e intergeneracional, el status quo (la situación de la familia pobre sin protección social) amenaza tanto las expectativas y oportunidades de los individuos que casi 
cualquier transferencia de recursos representa una mejora sustantiva, incluso si introducen tratamientos desiguales, juicios de desconfianza y conservan la desigualdad de las relaciones interpersonales. En otros términos, la urgencia de la situación de los integrantes de una familia pobre latinoamericana parece triunfar sobre (casi) cualquier consideración moral relevante.

\section{Conclusión}

El auge de las TMC en Latinoamérica se ha celebrado como un camino novedoso que los Estados adoptaron para promover la igualdad de oportunidades, la inclusión y la igualdad de género (ANSES 2012, pp. 17-30).

Este texto mostró que esa celebración es, como mínimo, apresurada y, como máximo, cínica. Sin duda, luego de años de inacción estatal, cualquier transferencia de recursos hacia los miembros menos aventajados constituye, por sí misma, una mejora no desdeñable respecto del status quo. Sin embargo, tal mejora no debería minimizar el hecho de que la justificación de las TMC supone una lógica de urgencia que termina por ser autocomplaciente con las obligaciones de bienestar de una comunidad política democrática.

Las TMC pueden diseñarse y ponerse en marcha de diversas maneras (Cecchini y Madariaga 2011, pp. 89-90): pueden incorporar a los propios beneficiarios como participantes activos en su evaluación y control (Adato y Roopnaraine 2010), pueden exigir condicionalidades leves que sean parcialmente negociadas con cada familia (Huneeus y Repetto 2013), pueden funcionar como instrumentos para que el Estado promueva la igualdad de género (Adato y Roopnaraine 2010), pueden promover relaciones igualitarias si la calidad de la educación pública es lo suficientemente atractiva para que las familias asalariadas envíen a sus hijos al mismo colegio que los beneficiarios de las TMC (Kerstenetzky 2009). La lógica de la urgencia es responsable de elegir, entre estas distintas aplicaciones y diseños posibles, aquellas que no tienen capacidad para construir relaciones igualitarias entre los ciudadanos.

Este texto mostró que esta lógica no puede abandonarse a menos que se debiliten en forma radical dos supuestos de las TMC: el que se vincula con el hecho de que la carga de la prueba se coloque del lado de las transferencias incondicionales y el que se relaciona con la inclusión de un criterio de responsabilidad individual atributiva. Dicho de otra manera, para elegir diseños y condicionalidades que promuevan relaciones igualitarias deben adoptarse otros principios normativos como

Diánoia, vol. LXII, no. 79 (noviembre de 2017). 
base de justificación; por ejemplo, estos programas no deberían limitar las obligaciones de protección de la comunidad con juicios controversiales acerca de cuán responsables son los individuos desaventajados de su comportamiento; no deberían fomentar y utilizar estándares de evaluación de conducta que se montan sobre la estabilidad de la sospecha y la desconfianza; por último, deberían realizar un esfuerzo por mostrar que su legitimidad no depende de razones controvertidas que se producen en un contexto desigual e inequitativo. ${ }^{9}$

\section{BIBLIOGRAFÍA}

Adato, M. y J. Hoddinott (comps.), 2010, Conditional Cash Transfers in Latin America, Johns Hopkins University Press, Baltimore, Maryland.

Adato, M. y T. Roopnaraine, 2010, "Women's Status, Gender Relations, and Conditional Cash Transfers", en Adato y Hoddinott 2010, pp. 284-314.

Anderson, E. 1999, "What is the Point of Equality?", Ethics, vol. 109, no. 2, pp. 287-337.

ANSES, 2012, La asignación universal por hijo para protección social en perspectiva. La política pública como restauradora de derechos, Administración Nacional de la Seguridad Social-Observatorio de la Seguridad Social, Buenos Aires.

Blake, M. y M. Risse, 2008, "Two Models of Equality and Responsibility", Canadian Journal of Philosophy, vol. 38, no. 2, pp. 165-199.

Bobonis, G.J. y F. Finan, 2009, "Neighborhood Peer Effects in Secondary School Enrollment Decisions", The Review of Economics and Statistics, vol. 91, no. 4, pp. 695-716.

Bohman, J., 1996, Public Deliberation, The MIT Press, Cambridge.

Cecchini, S. y A. Madariaga, 2011, Programas de transferencias condicionadas: balance de la experiencia reciente en América Latina y el Caribe, Naciones Unidas, Santiago de Chile.

Cecchini, S. y R. Martínez, 2011, Protección social inclusiva en América Latina. Una mirada integral, un enfoque de derechos, Naciones Unidas, Santiago de Chile.

Cruces, G., H. Rovner, y A. Schijman, 2007, "Percepciones sobre los Planes Sociales en Argentina", Banco Mundial (Documentos de Trabajo sobre Políticas Sociales 2), Buenos Aires.

De Brauw, A. y J. Hoddinott, 2011, "Must Conditional Cash Transfer Programs Be Conditioned to Be Effective? The Impact of Conditioning Transfers on School Enrollment in Mexico", Journal of Development Economics, vol. 96, no. 2, pp. 359-370.

Draibe, S.M., 2006, "Brasil: Bolsa-Escola y Bolsa-Família", en E. Cohen y R. Franco (comps.), Transferencias con corresponsabilidad: una mirada latino-

9 Agradezco a los árbitros de Diánoia por sus valiosos comentarios que permitieron mejorar la versión final de este trabajo. 
americana, Facultad Latinoamericana de Ciencias Sociales/Secretaría de Desarrollo Social, México, pp. 139-178.

Dworkin, G., 2013, “Defining Paternalism", en Ch. Coons y M. Weber (comps.), Paternalism: Theory and Practice, Cambridge University Press, Cambridge, pp. 25-38.

Fiszbein, A. y N. Schady, 2009, Transferencias monetarias condicionadas. Reducción de la pobreza actual y futura, trad. I. Caviedes Hoyos, Banco Mundial/Mayol, Washington.

Fourie, C., 2012, "What Is Social Equality? An Analysis of Status Equality as a Strongly Egalitarian Ideal", Res Publica, vol. 18, no. 2, pp. 107-126.

Freeland, N., 2007, "Superfluous, Pernicious, Atrocious and Abominable? The Case against Conditional Cash Transfers", IDS Bulletin, vol. 38, no. 3, pp. 75-78.

Gasparini, L. y G. Cruces, 2010, "Las asignaciones universales por hijo en Argentina: impacto, discusión y alternativas", Económica, vol. 56, pp. 105146.

González, M. e I. Tuñón, 2012, "Efecto de las políticas de transferencias condicionadas en la inclusión educativa", Revista Latinoamericana de Estudios Educativos, vol. 42, no. 4, pp. 33-53.

Huneeus, C. y C. Repetto, 2013, "Los desafíos pendientes del ingreso ético familiar", en J. Fantuzzi (comp.), Ingreso ético familiar: innovando en la lucha contra la pobreza, Libertad y Desarrollo, Santiago de Chile, pp. 219-251.

Hurley, S., 2003, Justice, Luck and Knowledge, Harvard University Press, Cambridge.

Kerstenetzky, C., 2009, "Redistribuição e desenvolvimento? A economia política do programa Bolsa Família", Revista Dados, vol. 52, no. 1, pp. 53-83.

Kymlicka, W., 2006, "Left-Liberalism Revisited", en Ch. Sypnowich (comp.), The Egalitarian Conscience. Essays in Honour of G.A. Cohen, Oxford University Press, Oxford, pp. 9-35

Lo Vuolo, R., 2011, "Los programas de transferencias monetarias condicionadas en América Latina y las perspectivas de la renta básica o ingreso ciudadano", Revista Internacional de Pensamiento Político, vol. 6, época I, pp. 193-222.

Long, R., 2016, "Luck Egalitarianism, Responsibility and Political Liberalism", Dialogue, vol. 55, pp. 107-130.

Maluccio, J., M. Adato y E. Skoufias, 2010, “Combining Quantitative and Qualitative Research Methods for the Evaluation of Conditional Cash Transfer Programs in Latin America", en Adato y Hoddinott 2010, pp. 26-52.

Matravers, M., 2003, "Responsibility and Choice", en M. Matravers (comp.), Scanlon and Contractualism, Frank Cass Publisher, Oregon, pp. 65-78.

Pautassi, L., P. Arcidiácono, y L. Royo, 2010, "Idas y vueltas en las políticas sociales: el protagonismo de la pobreza en la agenda política", en CELS. Derechos humanos en la Argentina. Informe 2010, Buenos Aires, Siglo XXI, pp. 223-261. 
Pautassi, L., P. Arcidiácono, y L. Royo, 2013, Asignación Universal por Hijo para la protección social de Argentina. Entre la satisfacción de necesidades y el reconocimiento de derechos, Naciones Unidas/Comisión Económica para América Latina y el Caribe/Fondo de las Naciones Unidas para la Infancia, Santiago de Chile.

Pérez Ribas, R., F. Veras Soares y G.I. Hirata, 2008, “The Impact of CCTs. What We Know and What We Are Not Sure About", Poverty in Focus, vol. 15, pp. 12-13.

Pierik, R., 2015, "Mandatory Vaccination: An Unqualified Defense”, General Subseries Research Paper no. 2015-02; Amsterdam Law School Research Paper no. 2015-08, disponible en $<$ http://ssrn.com/abstract $=2574634>$.

Pinzani, A., 2014, "Alcances e limites de um CCT Program: quão justificadas são as críticas ao Bolsa Família?", Cadernos de Filosofia Alemã: Crítica e Modernidade, vol. 19, no. 2, pp. 149-163.

Queralt, J., 2014, Igualdad, suerte y justicia, Marcial Pons, Madrid.

—, 2015, "El igualitarismo de la suerte", Doxa, vol. 38, pp. 189-213.

Rawls, J., 1993, Political Liberalism, Columbia University Press, Nueva York. , 1999, Theory of Justice. Revised Edition, Harvard University Press, Cambridge.

—_, 2004, La justicia como equidad. Una reformulación, trad. A. de Francisco, Paidós, Buenos Aires.

Scanlon, Th., 1998, What We Owe to Each Other, Harvard University Press, Cambridge, Mass.

—_, 2008, Moral Dimensions, Belknap Press of Harvard University Press, Cambridge, Mass.

Schady, N. y C. Araujo, 2008, "Cash Transfers, Conditions, School Enrollment, and Child Work: Evidence from a Randomized Experiment in Ecuador", Economía, vol. 8, no. 2, pp. 43-70.

Scheffler, S., 2003, "What Is Egalitarianism?", Philosophy and Public Affairs, vol. 31 , no. 1 , pp. 5-39.

- 2005, "Choice, Circumstance and the Value of Equality", Politics, Philosophy and Economics, vol. 4, no. 1, pp. 5-28.

Shiffrin, S., 2000, "Paternalism, Unconscionability Doctrine, and Accommodation", Philosophy and Public Affairs, vol. 29, no. 3, pp. 205-250.

Vargas, L., 2011, "Chile solidario: pasado y futuro de la vía chilena a la protección social (2003-2011)", Revista CIS, no. 14, pp. 23-44.

Veras Soares, F., R. Pérez Ribas y G.I. Hirata, 2008, "Los logros y las carencias de las transferencias de efectivo condicionadas: evaluación del impacto del Programa Tekoporã del Paraguay", International Poverty Centre Evaluation Note, no. 3, pp. 1-24.

Voorhoeve, A., 2008, "Scanlon on Substantive Responsibility", The Journal of Political Philosophy, vol. 16, no. 2, pp. 184-200.

Wolff, J., 1998, "Fairness, Respect and the Egalitarian Ethos", Philosophy and Public Affairs, vol. 27, no. 2, pp. 97-122. 